\title{
Association between two polymorphisms in the HLA-G gene and angiographic coronary artery disease
}

\author{
CHIARA BOIOCCHI ${ }^{1}$, SARA BOZZINI ${ }^{2}$, MICHELE ZORZETTO ${ }^{3}$, GABRIELE PELISSERO ${ }^{4}$, \\ MARIACLARA CUCCIA ${ }^{1}$ and COLOMBA FALCONE ${ }^{2,5}$
}

\author{
${ }^{1}$ Laboratory of Immunogenetics, Department of Genetics and Microbiology; ${ }^{2}$ Interdepartmental Center for Research \\ in Molecular Medicine (CIRMC); ${ }^{3}$ Laboratory of Biochemistry and Genetics, Institute for Respiratory Disease, \\ Foundation IRCCS Policlinico San Matteo, University of Pavia, Pavia; ${ }^{4}$ Scientific Direction IRCCS Policlinico San Donato, \\ Milan; ${ }^{5}$ Department of Cardiology, Istituto di Cura Città di Pavia University Hospital, Pavia, Italy
}

Received December 20, 2011; Accepted February 14, 2012

DOI: $10.3892 / \mathrm{mmr} .2012 .825$

\begin{abstract}
Atherosclerosis and related complications still represent the major cause of morbidity and mortality in industrialized countries. Therefore, it is particularly important to investigate the molecules involved in cardiac inflammation. Evidence exists showing that the human leukocyte antigen-G (HLA-G) gene tissue expression and related protein physiological significance is influenced by two polymorphisms, rs16375 and rs1632933. In this study, allelic, genotypic and haplotypic frequencies of a 14-bp insertion/deletion (Ins/Del) (rs16375) and of rs1632933 polymorphisms of the HLA-G gene were investigated in 664 patients with coronary artery disease (CAD) and 345 matched controls by polymerase chain reaction (PCR)-restriction fragment length polymorphism analysis and real-time PCR. The frequency of the Ins/Ins genotype was significantly higher in patients with CAD compared to the controls $(\mathrm{P}=0.018)$. After analysis of confounding variables, the results showed that the homozygous Ins/Ins was significantly and independently associated with the presence of angiographic CAD (odds ratio $2.09,95 \%$ confidence interval $1.10-4.02, \mathrm{P}=0.03$ ). Our data demonstrate a new risk factor for this multifactorial inflammatory disease.
\end{abstract}

\section{Introduction}

Atherosclerosis and related complications still represent the major cause of morbidity and mortality in industrialized countries. The mechanisms governing the progression and destabilization of atheromatous lesions are multiple and complex, involving immune and inflammatory reactions. In particular, the interactions of cells of the innate immune system

\footnotetext{
Correspondence to: Dr Chiara Boiocchi, Laboratory of Immunogenetics, Department of Genetics and Microbiology, University of Pavia, Via Ferrata 1, I-27100 Pavia, Italy

E-mail: chiara.boiocchi@unipv.it
}

Key words: human leukocyte antigen G, polymorphisms, coronary artery disease, immunogenetics and inflammatory mediators orchestrate aspects of endothelial injury and subsequent plaque formation (1-3).

The human leukocyte antigen-G (HLA-G) is a non-classic class I molecule of the major histocompatibility complex (MHC) (4). The basal expression of HLA-G is generally restricted to fetal tissues (5), adult thymic medulla (6), cornea (7), pancreatic islets (8) and, notably, endothelial cell precursors (9). It is worth noting, however, that HLA-G expression is upregulated in a number of inflammatory conditions (10-15). During the inflammatory response, HLA-G expressed by monocytes acts as a negative immune regulator (16-21), downregulating the production of Th1 as well as Th2 cytokines (22). This fact is significant for inflammation phenomena evolution.

There is evidence to suggest that the tissue expression of HLA-G may be influenced by genetic polymorphisms on the HLA-G gene $(23,24)$. In particular, a HLA-G insertion (Ins)/ deletion (Del) polymorphism (14 bp in exon 8 of the rs16375 gene) has been suggested to play a role in mRNA stability which affects HLA-G protein expression $(25,26)$. This polymorphism is linked with another polymorphism (rs1632933) of HLA-G. Previous studies (27-29) have provided evidence of a significant involvement of these polymorphisms in situations where immune dysregulation plays a crucial pathogenetic role, including several inflammatory-mediated human disorders.

Working under the assumption that atherosclerosis is a chronic inflammatory disease, and considering the presence of the HLA-G protein in human endothelial cell precursors, assessing the frequency of these polymorphic variants in patients with coronary artery disease (CAD) is of vital importance. In this study, we sought to achieve this goal focusing on patients scheduled for coronary angiography.

\section{Materials and methods}

Study subjects. A total of 664 Caucasian Italian patients with CAD (530 males, 114 females; mean age, $63.2 \pm 8$ years) were consecutively recruited from subjects referred for coronary angiography at the Cardiology Department of the University Hospital of Pavia, Italy. In each enrolled patient, a coronary lesion with $>50 \%$ luminal stenosis in at least one major coronary vessel was documented by coronary angiography. Patients 
were excluded if they had a previous myocardial infarction, prior coronary revascularization, inflammatory bowel disease, asthma, allergic disorders, human immunodeficiency virus infection, autoimmune or malignant disease, history of organ transplantation, renal, or liver disease. All the study participants had no evidence of peripheral artery or cerebrovascular disease.

A total of 345 healthy blood donors matched for age and gender were employed as the controls. All included subjects were Caucasians, ascertained to be of Italian descent. A complete clinical history was obtained from all participants. The study protocol was approved by the local Ethics Committee of the University of Pavia and written informed consent to participate was obtained from all subjects.

Definition of cardiac risk factors. Established risk factors were defined as follows: hypertensive patients were defined as those in whom blood pressure was $>140 / 90 \mathrm{mmHg}$ (confirmed by measurements on several occasions) or had a history of antihypertensive therapy. Hypercholesterolemia was considered to be present if the serum cholesterol was $>220 \mathrm{mg} / \mathrm{dl}$ or by the use of lipid lowering medications. Diabetes mellitus type 2 was defined as a prior diagnosis of the disease, a history of antidiabetic medication, or plasma fasting glucose levels of $>126 \mathrm{mg} / \mathrm{dl}$ on two or more occasions. Cigarette smoking was categorized into 'ever' or 'never', with ever smoking defined as having smoked $>3$ cigarettes/day for at least 1 year. A positive family history of coronary disease was defined as documented CAD in parents or siblings before the age of 60 years in men, and 70 years in women. Each of these risk factors was coded as a categorical variable as either present or absent.

Genetic analysis. We studied the polymorphisms, rs16375 and rs1632933, as they were identified through Haploview (http:// www.broadinstitute.org/haploview) to be the most significant polymorphisms for our research.

Genomic DNA was extracted from EDTA-treated blood using the GFX DNA purification kit (Amersham Biosciences, Piscataway, NJ, USA). Polymerase chain reaction (PCR) amplification of exon 8 of the HLA-G gene was performed as previously described (30). PCR amplifications were carried out in a total volume of $25 \mu \mathrm{l}$ containing: $1 \mu \mathrm{l}$ of genomic DNA (final concentration $20 \mathrm{ng} / \mu \mathrm{l}$ ), $0.1 \mu 1$ of $\mathrm{Taq}$ polymerase (Eurobio, Les Ulis, France; final concentration $0.02 \mathrm{U} / \mu \mathrm{l}), 1 \mu \mathrm{l}$ of each primer (final concentration $4 \mathrm{ng} / \mu \mathrm{l}$ ), $2.5 \mu \mathrm{l}$ 10X PCR buffer (Eurobio), $0.75 \mu 1 \mathrm{MgCl}_{2}$ (Eurobio) (final concentration $1.5 \mathrm{mM}$ ), $2.5 \mu \mathrm{l} \mathrm{dNTPs}$ (final concentration $2 \mathrm{mM}$ ). PCR reactions of the 14-bp Ins/Del polymorphism of the HLA-G gene were performed using a I-Cycler (BioRad, Monza, Italy) under the following conditions: initial incubation at $94^{\circ} \mathrm{C}$ for $2 \mathrm{~min}$, followed by 35 cycles each of $94^{\circ} \mathrm{C}$ for $30 \mathrm{sec}, 64^{\circ} \mathrm{C}$ for $1 \mathrm{~min}, 72^{\circ} \mathrm{C}$ for $2 \mathrm{~min}$; and a final extension at $72^{\circ} \mathrm{C}$ for $7 \mathrm{~min}$. The amplified PCR products were visualized on a $3 \%$ agarose gel stained with ethidium bromide. Amplicon sizes for the 14-bp polymorphism were $224 \mathrm{bp}$ for the presence of the 14-bp fragment and $210 \mathrm{bp}$ for the absence of the 14-bp fragment. PCR reactions of rs1632933 were performed using the LC480 thermocycler (Roche) by following Fret probes and primers (sensor, CTGTGATTACTGGGATCAG; anchor, CTGTGATT ACTAGGATCAG; primers: HLA-G R2, TGTCCTTAGCCAG
GGACCTT and HLA-G F2, TGTGACAATGAAGGACAGA TTTATAACCTT).

Statistical analysis. All statistical analyses were conducted with use of the SPSS statistical package version 11.0 (SPSS Inc., Chicago, IL, USA). The Kolmogorov-Smirnov test of normality was performed to verify the distribution of continuous data. A $\chi^{2}$ test for goodness of fit was used to verify whether the observed allele frequencies agreed with those expected under the HardyWeinberg equilibrium. Allelic and genotypic distributions were estimated by allele counting and compared in the CAD and control groups by the $\chi^{2}$ test. Univariate and multivariate logistic regression analyses were performed to examine whether the 14-bp Ins/Del polymorphism was associated with the risk of CAD after adjustment for age, gender and common cardiovascular risk factors. Crude and adjusted odds ratios (ORs) are reported with their 95\% confidence intervals (CIs). The study power was calculated using StatMate software, version 2.0 (GraphPad, San Diego, CA, USA). Two-tailed P-values $<0.05$ were deemed to indicate statistically significant differences.

Frequencies and haplotype reconstructions were calculated using the H PLUS program from http://cougar.fhcrc.org/hplus/. Linkage disequilibrium was calculated using the JLIN 1.6.0 software (http://www.genepi.org.au/jlin/).

\section{Results}

The general characteristics of the study participants are depicted in Table I. As expected, the prevalence of common cardiac risk factors was significantly higher in CAD patients than in the controls. The distribution of the HLA-G Ins/Del polymorphism is shown in Table II.

Genotypes were in the Hardy-Weinberg equilibrium both in the patients and controls. Based on the observed prevalence of the homozygous Ins/Ins genotype in the healthy population, our sample size had a $95 \%$ power to detect a relative risk of 2.10 for CAD between carriers and non-carriers with a significance level $(\alpha)$ of 0.05 (two-tailed). The frequency of the homozygous Ins/Ins genotype found in our study was significantly higher in patients with CAD (21.2\%) as compared to controls (15\%) $(\mathrm{P}=0.018)$. The crude $\mathrm{OR}$ for the presence of CAD in subjects bearing the homozygous Ins/Ins genotype was 1.51 (95\% CI 1.07-2.15; $\mathrm{P}=0.018)$.

To clarify whether the observed association was independent of potential confounders, we performed a multivariate logistic regression analysis after allowance for potential confounders, including all the common cardiac risk factors listed in Table I. After confounding variables were controlled, the results showed that the homozygous Ins/Ins was significantly and independently associated with the presence of angiographic CAD (OR 2.09, 95\% CI 1.10-4.02, $\mathrm{P}=0.03$ ). We analyzed a second polymorphism of HLA-G A/G (rs1632933). Our data showed no significant deviations between the 2 groups as regards either allelic or genotypic frequencies (Table III). After having analyzed the allelic and genotypic frequencies of 2 HLA-G polymorphisms, our attention was focused on the importance of haplotype reconstruction. From the analysis of haplotypic frequencies, we did not obtain a statistically significant difference of frequencies in the group of CAD patients vs. the controls (Fig. 1). Regarding the frequencies of the Del,A 
Table I. General characteristics of the study participants.

\begin{tabular}{lccr}
\hline Characteristic & CAD patients (n=664) & Controls (n=345) & P-value \\
\hline Age, years & $63.2 \pm 8.0$ & $64.1 \pm 10.8$ & 0.520 \\
Male gender, n (\%) & $530(80)$ & $250(73)$ & 0.200 \\
Type 2 diabetes (\%) & $119(15)$ & $13(4)$ & $<0.010$ \\
Hypertension (\%) & $381(57)$ & $27(9)$ & $<0.001$ \\
Smokers (\%) & $252(38)$ & $22(6)$ & $<0.001$ \\
Hypercholesterolemia (\%) & $173(26)$ & $18(5)$ & $<0.001$ \\
Family history of CAD (\%) & $143(21)$ & $15(4)$ & $<0.001$
\end{tabular}

P-values were calculated by means of the Student's t-test or $\chi^{2}$ test, as appropriate. CAD, coronary artery disease.

Table II. Distribution of HLA-G genotypes in the study participants.

\begin{tabular}{lccc}
\hline Genotype & CAD (n=664) & Controls (n=345) & OR (95\% CI) \\
\hline Ins/Ins (\%) & $141(21.2)$ & $52(15)$ & $1.51(1.07-2.15)$ \\
Ins/Del plus Del/Del (\%) & $523(78.8)$ & $293(85)$ & Reference
\end{tabular}

P-values were calculated by means of the $\chi^{2}$ test. CAD, coronary artery disease; OR, odds ratio; CI, confidence interval; Ins, insertion; Del, deletion.

Table III. Distribution of HLA-G genotypes of Rs1632933 polymorphism in the study participants.

\begin{tabular}{lccc}
\hline Genotype & CAD $(\mathrm{n}=664)$ & Controls $(\mathrm{n}=345)$ & OR (95\% CI) \\
\hline A/A $(\%)$ & $165(25)$ & $95(27)$ & $1.0172(0.7478-1.3838)$ \\
A/G plus G/G $(\%)$ & $499(75)$ & $250(72)$ & Reference
\end{tabular}

CAD, coronary artery disease; OR, odds ratio; CI, confidence interval.

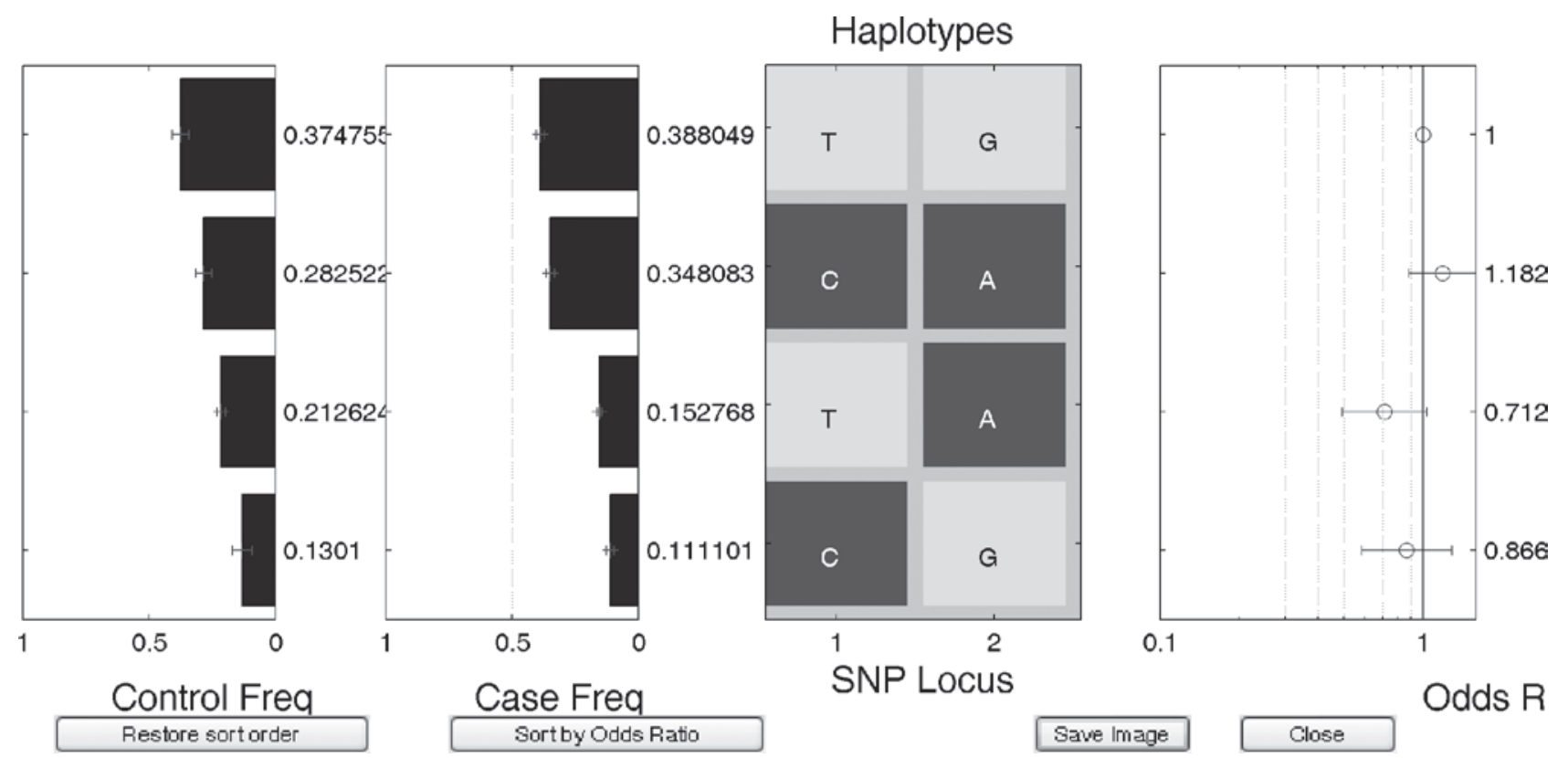

Figure 1. Frequencies of HLA-G (rs16375 and rs1632933) haplotypes in patients with coronary artery disease and healthy controls. The deletion (Del) allele of the 14-bp (insertion) Ins/Del polymorphism of HLA-G is indicated with letter T. The allele Ins of 14-bp Ins/Del polymorphism of HLA-G is indicated with letter C. Freq, frequency; Odds R, odds ratio. 
haplotype, the results were not statistically significant (OR 0.71 , $95 \%$ CI $0.49 \pm 1.03, \mathrm{P}=0.072$ ).

\section{Discussion}

To the best of our knowledge, the present study is the first to demonstrate that the homozygous Ins/Ins genotype of the HLA-G 14-bp Ins/Del polymorphism (rs16375) is significantly associated with the presence of CAD in the Italian population. Notably, this association persisted after adjustments for potential confounders, including common cardiac risk factors as shown by the multivariate logistic regression analysis.

Growing evidence has now accrued that the 14-bp polymorphism in the HLA-G gene is associated with splicing variants and the stability pattern of HLA-G mRNA $(25,26)$. In particular, the insertion of this 14-bp sequence has been linked to a decreased mRNA stability resulting in lower circulating levels of the anti-inflammatory soluble HLA-G molecule (sHLA-G) 2 (6,31).

The results obtained in the present study show significant differences in the distribution of the HLA-G 14-bp polymorphism between Italian CAD patients and healthy subjects: the CAD population was characterized by an increased frequency of subjects with a genetically determined diminished production of the anti-inflammatory HLA-G molecule. From this first report of association between the 14-bp polymorphism in exon 8 of the HLA-G gene and CAD, it cannot be excluded that the association may be a consequence of linkage disequilibrium with other gene loci in the human MHC region. It is well described that the extended HLA-haplotype, including the HLA-DRB1*01/*04 combination, is associated with cardiovascular disease and mortality (32). Of interest, an association between HLA-G polymorphisms and other chronic inflammatory disorders, such as inflammatory bowel disease $(33,34)$, asthma $(35,36)$ and multiple sclerosis $(37-39)$, has been repeatedly reported. Besides the potential role played by HLA-G in inflammation and immunity (15), there is evidence to suggest that this molecule may also modulate endothelial cell activity $(40,41)$. In this regard, it has been previously demonstrated that purified recombinant soluble HLA-G is capable of downregulating endothelial cell proliferation and migration in vitro (42). Taken together, these data point to a subtle, but definite role of HLA-G in vascular homeostasis that warrants further research for its potential pathophysiological role in atherogenesis.

In interpreting our findings, important limitations must be considered. Firstly, our study was a single-center case-control study, and additional investigations with a larger number of patients would be useful to confirm the importance of this polymorphism in affecting the risk of CAD. However, our sample size was appropriately powered based upon the observed frequency of the homozygous Ins/Ins genotype in our control population. Secondly, the link between the HLA-G polymorphism and CAD was observed in a group of Caucasian subjects from Italy. Therefore, further studies are required to clarify its association with CAD in different ancestral populations. Finally, in our study we did not specifically measure the levels of sHLA-G in the sera of our study participants; however, as the levels of sHLA-G are known to change according to different inflammatory responses, it would be difficult to correlate sHLA-G with CAD.
In conclusion, the data from our present study on the significant association between the HLA-G Ins/Ins genotype and $\mathrm{CAD}$ add a new risk factor to the multifactorial genetic contribution to this common inflammatory disease. We are the first group to have studied the 14-bp Ins/Del polymorphism of HLA-G in a CAD population. Future investigations should be focused on understanding the exact role of HLA-G genetic variants in the atherosclerotic processes, possibly by focusing on the functional association between the possibly reduced sHLA-G production and the 14-bp HLA-G polymorphism. In the future, we will analyze other important risk factors for the CAD development, such as $\mathrm{C}$ reactive protein, cytokines and fibrinogene.

\section{References}

1. Lusis AJ: Atherosclerosis. Nature 407: 233-234, 2000.

2. Ross R: Atherosclerosis is an inflammatory disease. Am Heart J 138: S419-S420, 1999.

3. Koenig W: Inflammation and coronary heart disease: an overview. Cardiol Rev 9: 31-35, 2001.

4. Heinrichs H and Orr HAT: HLA non-A,B,C class I genes: their structure and expression. Immunol Res 9: 265-274, 1990.

5. Redman CW, McMichael AJ, Stirrat GM, et al: Class I major histocompatibility complex antigens on human extravillous cytotrophoblast. Immunology 52: 457-468, 1984.

6. Crisa L, McMaster MT, Ishii JK, et al: Identification of a thymic epithelial cell subset sharing expression of the class Ib HLA-G molecule with fetal trophoblasts. J Exp Med 186: 289-298, 1997.

7. Le Discorde M, Moreau P, Sabatier P, et al: Expression of HLA-G in human cornea, an immuneprivileged tissue. Hum Immunol 64: 1039-1044, 2003.

8. Cirulli V, Zalatan J, McMaster M, et al: The class I HLA repertoire of pancreatic islets comprises the nonclassical class $\mathrm{Ib}$ antigen HLA-G. Diabetes 55: 1214-1222, 2006.

9. Hiby SE, King A, Sharkey A and Loke YW: Molecular studies of trophoblast HLA-G: polymorphism, isoforms, imprinting and expression in preimplantation embryo. Tissue Antigens 53: 1-13, 1999.

10. Wiendl H, Behrens L, Maier S, et al: Muscle fibers in inflammatory myopathies and cultured myoblasts express the nonclassical major histocompatibility antigen HLA-G. Ann Neurol 48: 679-684, 2000.

11. Aractingi S, Briand N, Le Danff C, et al: HLA-G and NK receptor are expressed in psoriatic skin: a possible pathway for regulating infiltrating T cells? Am J Pathol 159: 71-77, 2001.

12. Wiendl H, Feger U, Mittelbronn M, et al: Expression of the immune-tolerogenic major histocompatibility molecule HLA-G in multiple sclerosis: implications for CNS immunity. Brain 128: 2689-2704, 2005.

13. Carosella ED, Paul P, Moreau P and Rouas-Freiss N: HLA-G and HLA-E: fundamental and pathophysiological aspects. Immunol Today 21: 532-534, 2000.

14. Carosella ED, Moreau P, Lemaoult J and Rouas-Freiss N: HLA-G: from biology to clinical benefits. Trends Immunol 29: 125-132, 2008.

15. Baricordi OR, Stignani M, Melchiorri L and Rizzo R: HLA-G and inflammatory diseases. Inflamm Allergy Drug Targets 7: 67-74, 2008.

16. Hofmeister V and Weiss EH: HLA-G modulates immune responses by diverse receptor interactions. Semin Cancer Biol 13: 317-323, 2003.

17. Bainbridge DR and Ellis SA: HLA-G suppresses proliferation of CD4 T-lymphocytes. J Reprod Immunol 48: 17-26, 2000.

18. Lila N, Rouas-Freiss N, Dausset J, et al: Soluble HLA-G protein secreted by allo-specific $\mathrm{CD} 4^{+} \mathrm{T}$ cells suppresses the alloproliferative response: a CD4 ${ }^{+} \mathrm{T}$ cell regulatory mechanism. Proc Natl Acad Sci USA 98: 12150-12155, 2001.

19. Le Gal FA, Riteau B, Sedlik C, et al: HLA-G-mediated inhibition of antigen-specific cytotoxic T lymphocytes. Int Immunol 11: 1351-1356, 1999.

20. Bainbridge DR, Ellis SA and Sargent I: HLA-G suppresses proliferation of CD4(+) T-lymphocytes. J Reprod Immunol 48: 17-26, 2000. 
21. Contini P, Ghio M, Merlo A, et al: Apoptosis of antigen-specific $\mathrm{T}$ lymphocytes upon the engagement of CD8 by soluble HLA class I molecules is Fas ligand/Fas mediated: evidence for the involvement of p56lck, calcium calmodulin kinase II, and Calcium-independent protein kinase $\mathrm{C}$ signaling pathways and for NF-kappaB and NF-AT nuclear translocation. J Immunol 175: 7244-7254, 2005.

22. Feger U, Tolosa E, Huang YH, et al: HLA-G expression defines a novel regulatory $\mathrm{T}$-cell subset present in human peripheral blood and sites of inflammation. Blood 110: 568-577, 2007.

23. Harrison GA, Humphrey KE, Jakobsen IB and Cooper DW: A 14-bp deletion polymorphism in the HLA-G gene. Hum Mo Genet 2: 2200, 1993

24. Van der Ven K, Skrablin S, Engels G and Krebs D: HLA-G polymorphisms and allele frequencies in Caucasians. Hum Immuno 59: 302-312, 1998.

25. Rousseau P,Le Discorde M, Mouillot G, et al: The 14 bp deletioninsertion polymorphism in the 3' UT region of the HLA-G gene influences HLA-G mRNA stability. Hum Immunol 64 1005-1010, 2003

26. Hviid TV, Hylenius S, Rørbye C and Nielsen LG: HLA-G allelic variants are associated with differences in the HLA-G mRNA isoform profile and HLA-G mRNA levels. Immunogenetics 55: $63-79,2003$

27. Gazit E, Slomov Y, Goldberg I, et al: HLA-G is associated with Pemphigus vulgaris in Jewish patients. Hum Immunol 65: 39-46, 2004

28. Glas J, Török HP, Tonenchi L, et al: The 14-bp deletion polymorphism in the HLA-G gene displays significant differences between ulcerative colitis and Crohn's disease and is associated with leocecal resection in Crohn's disease. Int Immunol 19: 621-626, 2007

29. Veit TD, Vianna P, Scheibel I, et al: Association of the HLA-G 14-bp insertion/deletion polymorphism with juvenile idiopathic arthritis and rheumatoid arthritis. Tissue Antigens 71: 440-444, 2009.

30. Hviid TV, Hylenius S, Hoegh AM, et al: HLA-G polymorphisms in couples with recurrent spontaneous abortions. Tissue Antigens 60: 122-132, 2002.
31. Rebmann V, van der Ven K, Passler M, et al: Association of soluble HLA-G plasma levels with HLA-G alleles. Tissue Antigens 57: 15-21, 2001

32. Farragher TM, Goodson NJ, Naseem H, et al: Association of the HLA-DRB1 gene with premature death, particularly from cardiovascular disease, in patients with rheumatoid arthritis and inflammatory polyarthritis. Arthritis Rheum 58: 359-369, 2008.

33. Torres MI, Le Discorde M, Lorite P, et al: Expression of HLA-G in inflammatory bowel disease provides a potential way to distinguish between ulcerative colitis and Crohn's disease. Int Immunol 16: 579-583, 2004.

34. Downs-Kelly E, Schade AE and Hansel DE: The role of HLA-G in gastrointestinal inflammatory disease and malignancy. Semin Cancer Biol 17: 451-458, 2007.

35. Nicolae D, Cox NJ, Lester LA, et al: Fine mapping and positional candidate studies identify HLA-G as an asthma susceptibility gene on chromosome 6p21. Am J Hum Genet 76: 349-357, 2005.

36. Rizzo R, Mapp CE, Melchiorri L, et al: Defective production of soluble HLA-G molecules by peripheral blood monocytes in patients with asthma. J Allergy Clin Immunol 115: 508-513, 2005.

37. Maier S, Geraghty DE and Weiss EH: Expression and regulation of HLA-G in human glioma cell lines. Transplant Proc 31: 1849-1853, 1999

38. Wiendl H: HLA-G in the nervous system. Hum Immunol 68 : 286-293, 2007

39. Fainardi E, Rizzo R, Melchiorri L, et al: Presence of detectable levels of soluble HLA-G molecules in CSF of relapsing-remitting multiple sclerosis: relationship with CSF soluble HLA-I and IL-10 concentrations and MRI findings. J Neuroimmunol 142: 149-158, 2003.

40. Dorling A, Monk N and Lechler R: HLA-G inhibits the transendothelial migration of human NK cells. Eur J Immunol 30: 586-593, 2000

41. Forte P, Pazmany L, Matter-Reissmann UB, et al: HLA-G inhibits rolling adhesion of activated human NK cells on porcine endothelial cells. J Immunol 167: 6002-6008, 2001.

42. Le Bouteiller P, Pizzato N, Barakonyi A and Solier C: HLA-G, pre-eclampsia, immunity and vascular events. J Reprod Immunol 59: 219-234, 2003. 